\title{
BMJ Open Comparison of the periodontal condition in Korean and Japanese adults: a cross-sectional study
}

\author{
Michiko Furuta, ${ }^{1}$ Kenji Takeuchi, ${ }^{1,2}$ Yoshihiro Shimazaki, ${ }^{3}$ Toru Takeshita, ${ }^{1,2}$ \\ Yukie Shibata, ${ }^{1}$ Jun Hata, ${ }^{4,5,6}$ Daigo Yoshida, ${ }^{4}$ Deok-Young Park, ${ }^{7}$ \\ Toshiharu Ninomiya, ${ }^{4,5}$ Yoshihisa Yamashita $^{1}$
}

To cite: Furuta M, Takeuchi K, Shimazaki Y, et al. Comparison of the periodontal condition in Korean and Japanese adults: a cross-sectional study. BMJ Open 2018;8:e024332. doi:10.1136/ bmjopen-2018-024332

- Prepublication history and additional material for this paper are available online. To view these files, please visit the journal online (http://dx.doi. org/10.1136/bmjopen-2018024332).

Received 22 May 2018 Revised 3 August 2018 Accepted 2 October 2018

Check for updates

(C) Author(s) (or their employer(s)) 2018. Re-use permitted under CC BY-NC. No commercial re-use. See rights and permissions. Published by BMJ.

For numbered affiliations see end of article.

Correspondence to Dr Yoshihisa Yamashita; yoshi@dent.kyushu-u.ac.jp

\section{ABSTRACT}

Objectives Reports from national surveys in South Korea and Japan have indicated that the prevalence of periodontal disease is lower in Korea than in Japan. However, these national surveys have not evaluated factors related to periodontal health condition, including diabetes and metabolic syndrome. This study compared periodontal conditions between Korean and Japanese adults, in the context of general health status.

Design Cross-sectional study.

Setting National survey in South Korea (Korean National Health and Nutrition Examination Survey, 'KNHANES') and a population-based study in Japan (Hisayama study); both were conducted in 2012.

Participants This study included 3574 Korean and 2205 Japanese adults aged 40-79 years.

Outcome measures Periodontal condition was assessed by using the Community Periodontal Index (CPI). Examiners in Japan underwent clinical calibration training for periodontal examination with a gold-standard examiner from KNHANES, prior to the Hisayama study.

Results The age-adjusted prevalences of periodontal disease, defined as CPI score $\geq 3$, were $31.4 \%$ and $42.1 \%$ in South Korea and Japan, respectively $(p<0.001)$. The age-adjusted prevalences of diabetes $(p=0.018)$ and metabolic syndrome $(p=0.001)$ were higher in Korea than in Japan. The numbers of present and filled teeth and percentages of participants who visited a dental clinic in the last 12 months were higher in Japan than in Korea (all $p<0.001)$. Logistic regression analysis showed that the Japanese participants were more likely to have periodontal disease than were the Korean participants, after adjusting for age, sex, occupation, oral health status, oral health behaviour, diabetes and metabolic syndrome.

Conclusions A higher prevalence of periodontal disease was found in Japanese participants than in Korean participants. Further studies are needed to more clearly elucidate factors underlying the difference in periodontal conditions between the two populations, including those related to the dental healthcare system and dietary intake.

\section{INTRODUCTION}

According to WHO Global Oral Health Data Bank, ${ }^{1}$ the prevalence of periodontal disease, as defined by the Community Periodontal Index (CPI) code $\geq 3$, was 4\%-37\%

\section{Strengths and limitations of this study}

- This is the first study to compare periodontal disease between Korean and Japanese people, in relation to systemic diseases.

- This study included a large representative population in Korea, based on a national survey and a community-based population in Japan.

- The examiners in the Japanese survey underwent clinical calibration training for the periodontal examination with a gold-standard examiner from the Korean survey.

- Periodontal condition was evaluated by using partial mouth assessment; thus, it was possible to underestimate the prevalence of periodontal disease.

in $35-44$ years and $20 \%-52 \%$ in $65-74$ years, among Asian countries (including the least developed to the highly industrialised) that conducted national surveys from 1999 to 2012. The general health conditions and healthcare systems vary widely among countries in Asia, depending on their levels of economic development. Oral health conditions and oral healthcare systems in this region also vary widely. ${ }^{2}$ Thus, differences in socioeconomic status might contribute to inequalities in oral health among Asian countries.

Surprisingly, the results of national surveys in Korea and Japan indicate that the prevalence of periodontal disease is lower in Korea (32.9\% and $23.9 \%$ of individuals aged 19 and over in 2008 and 2010, respectively) ${ }^{3}$ than in Japan $(44.7 \%$ and $41.5 \%$ of individuals aged 19 and over in 2005 and 2011, respectively). ${ }^{4}$ Among Asian countries, South Korea (hereafter 'Korea') and Japan are located in close proximity; they share similar economic and ethnic backgrounds. The gross domestic product per capita in 2016 was $\$ 27534$ in Korea and \$38895 in Japan. ${ }^{5}$ The Korean and Japanese populations belong primarily to the 
northern Mongoloid group, and Koreans are genetically closer to the Japanese than to other Mongoloid groups. ${ }^{6}$

With regard to healthcare systems, the governments of both Korea and Japan provide national health insurance that covers the entire population. In Korea, dental treatment covered by health insurance is limited; however, in recent years, dental insurance coverage has expanded, and denture treatment in individuals aged 65 and over (from 2016) and periodontal scaling (from 2013) has been covered. ${ }^{7}$ In contrast, in Japan, national health insurance covers almost all dental treatments, such as treatment for dental caries, including endodontic treatment, periodontal disease, prosthetic treatment and extraction. Periodontal treatment in Japan has been covered by health insurance since 1972. The size of the dental workforce is larger in Japan than in Korea; population ratios of dentists were reported as 5.0 and 7.4 per 10000 people in Korea and Japan, respectively. ${ }^{8}$ Additionally, the population ratios of practising dental hygienists were 4.9 and 8.5 per 10000 people in Korea and Japan, respectively. ${ }^{9}$ These data suggest that the Japanese may have an increased likelihood of receiving periodontal treatment and preventing care compared with Koreans, considering the greater dental insurance coverage and higher numbers of dentists and dental hygienists. Differences in healthcare systems between Korean and Japan seem to contradict the levels of periodontal health in these two countries.

However, great significance cannot be attached to the reports of these national surveys, as potential risk factors related to periodontal disease are not considered. Growing evidence from epidemiological studies indicates that diabetes and metabolic syndrome (MetS) are associated with periodontal disease. ${ }^{10}{ }^{11}$ When comparing periodontal disease among different countries, the effects of systemic health on periodontal disease and other risk factors should be addressed. Thus, a comparison of geographical and racial differences in periodontal disease, and interpretations of the reasons for these differences are essential for preventing periodontal disease. The objective of this study was to investigate whether periodontal condition differed between Korean and Japanese adults, in relation to the effects of diabetes and MetS.

\section{METHODS}

\section{Study population}

In the present study, data for Koreans were collected from a subset of the Fifth Korean National Health and Nutrition Examination Survey (KNHANES), a nationwide cross-sectional survey conducted in 2012 by the Korea Center for Disease Control and Prevention (CDC). Participants were selected by using a multistage clustered probability design to produce a nationally representative non-institutionalised civilian sample. Additional information regarding the KNHANES can be found elsewhere. ${ }^{12}$ The present study included 3955 participants from the 2012 survey, aged 40-79 years, who received medical and dental examinations in addition to a health interview. After excluding participants with missing data, 3574 participants were included in the analysis.

Data for Japanese were obtained from the Hisayama study, a population-based prospective study of cerebrocardiovascular diseases performed in the town of Hisayama (a suburban rural area of Fukuoka city in southern Japan). The age and occupational distributions of the population of this town are approximately representative of Japan as a whole. ${ }^{13}$ A portion of the Hisayama study in 2012 comprised dental examinations for residents aged $\geq 40$ years. The present study included 2342 participants, aged 40-79 years, who received both medical and dental examinations in 2012 (57.8\% of the total population in this age group). After excluding participants with missing data, 2205 participants were included in the analysis.

\section{Assessment of periodontal condition and dental caries}

Periodontal condition was assessed by using the CPI in both KNHANES and Hisayama studies. In accordance with WHO protocols, 10 teeth were selected for the periodontal examination: two molars in each posterior sextant, and the upper right and lower left central incisors. If no index tooth was present in a qualified sextant, all remaining teeth in that sextant were examined. The highest code among the examined sextants was recorded, to represent the CPI status for each individual. Periodontal disease was defined as CPI code $\geq 3$ (periodontal pocket depth $\geq 4 \mathrm{~mm}$ ).

Trained and calibrated dentists assessed periodontal conditions in both KNHANES and Hisayama studies. To ensure the reliability of measurements with CPI, seven examiners in the Hisayama study were calibrated to a gold-standard examiner in the 2012 KNHANES. The calibration was performed by conducting examinations on 10 Japanese volunteers who had all six sextants examined. The mean $\kappa$ value (range) between examiners in the Hisayama study and the gold-standard examiner in the 2012 KNHANES was 0.55 (0.43-0.66) for CPI code.

We assessed dental caries by measuring the total numbers of decayed and filled teeth; this measurement was performed because tooth surface roughness, especially in cases of subgingival restoration, leads to the accumulation of plaque, which results in gingival inflammation. ${ }^{14}$ Teeth were regarded as filled teeth if they had undergone permanent restoration (because of previous decay or other reasons) with a fixed dental prosthesis abutment.

\section{Assessment of diabetes and MetS}

A blood sample was collected from the antecubital vein in the morning, after fasting for at least 8 hours in the KNHANES, and after overnight fasting in the Hisayama study. Fasting plasma concentrations of glucose, triglycerides and high-density lipoprotein (HDL) cholesterol were measured. Revised HDL values were used in the KNHANES, because HDL values in the same samples differed between the Korean Central Laboratory and 
the $\mathrm{CDC}$ in the USA; conversion rates were calculated by analysing serum samples sent to the CDC, using the isotope dilution gas chromatography-mass spectrometry; this method is considered the gold standard. ${ }^{15}$ Diabetes was defined as fasting glucose $\geq 126 \mathrm{mg} / \mathrm{dL}$, or undergoing treatment for diabetes with medication and/or insulin injections. ${ }^{16}$ For the 2012 KNHANES group, haemoglobin A1c was solely assessed for individuals with diabetes or hyperglycaemia (fasting glucose $\geq 126 \mathrm{mg} / \mathrm{dL}$ ), and the oral glucose tolerance test was not conducted; therefore, the diagnostic criteria of diabetes used in this study did not correspond with the current diagnostic criteria proposed by the American Diabetes Association. MetS was defined on the basis of a Joint Interim Statement, using the criteria for Asians ${ }^{17}$ : elevated waist circumference ( $\geq 90 \mathrm{~cm}$ in men and $\geq 80 \mathrm{~cm}$ in women), elevated fasting glucose levels $(\geq 100 \mathrm{mg} / \mathrm{dL}$ or drug treatment for elevated glucose), elevated triglycerides $(\geq 150 \mathrm{mg}$ / $\mathrm{dL}$ or drug treatment for elevated triglycerides), reduced HDL $(<40 \mathrm{mg} / \mathrm{dL}$ in men and $<50 \mathrm{mg} / \mathrm{dL}$ in women) and elevated blood pressure (systolic blood pressure $\geq 130 \mathrm{~mm}$ $\mathrm{Hg}$ or diastolic blood pressure $\geq 85 \mathrm{~mm} \mathrm{Hg}$, or antihypertensive drug treatment). Individuals who were positive for three or more of these components were considered to have MetS.

\section{Measurement of other factors}

Information regarding participants' smoking habits, occupational statuses and dental visits in the last 12 months was obtained from a health interview in the KNHANES and from a self-administered questionnaire in the Hisayama study. Smokers were categorised as current, never or former smokers. Socioeconomic status was determined on the basis of occupational status, which was divided into four categories: clerical support workers; elementary occupations and unskilled workers; other jobs and home maker, unemployed or retired. 'Other jobs' includes professional, skilled and service workers, salespeople, farmers and fishers. We used occupation as a socioeconomic status indicator; however, we did not directly assess income because the Hisayama study did not collect income data and the previous KNHANES study (2012) showed no significant association between income and periodontal disease. ${ }^{18}$

\section{Statistical analysis}

The t-test or $\chi^{2}$ test was used to assess differences in the distribution of age, sex and job classification between the KNHANES and Hisayama studies. Age-adjusted mean values of the numbers of present teeth, decayed teeth and filled teeth, defined as quantitative variables, were estimated by using analysis of covariance. The proportions of periodontal disease, diabetes, MetS, number of teeth and oral health behaviours, defined as categorical variables, were adjusted on the basis of age distribution of WHO standard population in 2000-2025, by using the direct method. Differences in the mean values and proportions between countries were tested by linear regression modelling and logistic regression analysis, respectively. The association between periodontal disease (dependent variable) and country (independent variable) was assessed by using logistic regression analysis. We included participants who had more than 10 teeth in this analysis because all of them had at least one sextant in CPI. The association between number of teeth (dependent variable) and country (independent variable) was investigated by using generalised linear models with a Poisson probability distribution. Logistic regression models and generalised linear models were tested: model 1 included diabetes; model 2 included MetS and model 3 included individual MetS components. SEs were estimated by using robust or sandwich variance estimators of the covariance matrix of the regression coefficients, to correct for heteroskedasticity across countries. OR, $\beta$ coefficients and 95\% CI derived from corrected SEs were calculated. The impact of an unmeasured confounder on the estimated OR was examined in sensitivity analyses, based on Lin $e t$ $a l .{ }^{19}$ SPSS software (V.19.0 for Windows; IBM SPSS) and R V.3.2.1 were used for data analyses. $\mathrm{p}<0.05$ was considered statistically significant in all analyses.

\section{Patient and public involvement}

KNHANES data used in this study comprise nationwide data, which is collected annually by the Korea CDC; these data are publicly available to researchers. Participants in the Hisayama study were recruited with the help of the Health C\&C Center Hisayama, which provided healthcare services to the residents of Hisayama. None of our participants were involved in the recruitment or conduct of either study that has provided data for this report.

\section{RESULTS}

Demographic characteristics of the study participants in Korea and Japan are shown in table 1. Participants in Korea were significantly younger than those in Japan. Using age-adjusted means and the frequencies of dental and systemic parameters, it was observed that the percentage of participants with periodontal disease and the mean numbers of present and filled teeth were significantly higher in Japan than in Korea (table 2). The percentages of participants with diabetes and MetS were significantly higher in Korea than in Japan. In terms of individual MetS components, participants in Japan had a higher prevalence of elevated fasting glucose and blood pressure, and a lower prevalence of elevated triglycerides and reduced HDL, compared with those in Korea.

Logistic regression analyses showed that the Japanese participants were more likely to have periodontal disease than were the Korean participants (OR 1.68, 95\% CI 1.41 to 2.00); this was consistent even when diabetes and other covariates, including age, sex, number of teeth, number of decayed and filled teeth, dental visits, smoking, and occupation, were included in the model (table 3). Similar results were observed in models that included MetS and individual MetS components. 
Table 1 Demographic characteristics of Korean and Japanese participants aged $40-79$ years

\begin{tabular}{|c|c|c|c|}
\hline & Korean & Japanese & \multirow[b]{2}{*}{$P$ values } \\
\hline & $n=3574$ & $n=2205$ & \\
\hline Age & $58.4 \pm 11.0$ & $60.5 \pm 10.7$ & $<0.001$ \\
\hline Age category & & & $<0.001$ \\
\hline $40-49$ & 902 (25.2) & $434(19.7)$ & \\
\hline $50-59$ & $1009(28.2)$ & 514 (23.3) & \\
\hline $60-69$ & 936 (26.2) & $743(33.7)$ & \\
\hline $70-79$ & 727 (20.3) & 514 (23.3) & \\
\hline Sex & & & 0.074 \\
\hline Men & $1527(42.7)$ & $995(45.1)$ & \\
\hline Women & 2047 (57.3) & $1210(54.9)$ & \\
\hline Job classification & & & $<0.001$ \\
\hline $\begin{array}{l}\text { Clerical support } \\
\text { workers }\end{array}$ & $191(5.3)$ & 526 (23.9) & \\
\hline $\begin{array}{l}\text { Elementary } \\
\text { occupations }\end{array}$ & $394(11.0)$ & $128(5.8)$ & \\
\hline Other jobs & $1514(42.4)$ & 389 (17.6) & \\
\hline $\begin{array}{l}\text { Unemployed, } \\
\text { home makers and } \\
\text { part-time workers }\end{array}$ & 1475 (41.3) & 1162 (52.7) & \\
\hline
\end{tabular}

\section{DISCUSSION}

This study showed that periodontal disease was more prevalent in Japanese participants than in Korean participants, even after adjustment for diabetes and MetS. Our results suggest that this difference in prevalence was not related to the participants' systemic conditions. The strength of our study lies in the fact that the examiners in Japan underwent calibration for periodontal examination with a gold-standard examiner from the KNHANES, which helped to reduce bias in the prevalence estimates.

With regard to the results of the logistic regression models, the proportion of variance explained by the model adjusted for diabetes and covariates was relatively low (Nagelkerke's R-squared 13.6\%). This suggests that unmeasured factors might be associated with the differences in periodontal condition between Korean and Japanese participants. In post hoc sensitivity analyses, the association between country and periodontal disease was not significant in the logistic model; it was significant solely when there was a significant difference in the proportions of an unmeasured factor between Korean and Japanese participants (online supplementary table $1)$. In this model, periodontal disease could possibly be explained in relation to unmeasured factors, rather than in relation to country, which suggests that unmeasured factors contribute to the differences in periodontal condition.

Several factors, such as oral microbiota and dietary intake, could be the unmeasured factors that may have
Table 2 Age-adjusted mean values and frequencies of dental and systemic parameters by country

\begin{tabular}{|c|c|c|c|}
\hline & Korean & Japanese & $P$ values \\
\hline $\begin{array}{l}\text { Periodontal disease (CPI } \\
\text { code } \geq 3)^{\star}\end{array}$ & $31.4 \%$ & $42.1 \%$ & $<0.001$ \\
\hline No of teeth & $22.9 \pm 0.1$ & $24.1 \pm 0.1$ & $<0.001$ \\
\hline 0 & $2.0 \%$ & $1.0 \%$ & \\
\hline $1-9$ & $4.6 \%$ & $2.6 \%$ & \\
\hline $10-19$ & $8.5 \%$ & $7.6 \%$ & \\
\hline $20-27$ & $39.9 \%$ & $45.8 \%$ & \\
\hline$\geq 28$ & $45.0 \%$ & $42.9 \%$ & \\
\hline No of decayed teeth & $0.59 \pm 0.02$ & $0.59 \pm 0.03$ & 0.882 \\
\hline No of filled teeth & $7.0 \pm 0.1$ & $13.0 \pm 0.2$ & $<0.001$ \\
\hline $\begin{array}{l}\text { Dental visit in the last } \\
12 \text { months }\end{array}$ & $25.6 \%$ & $30.4 \%$ & $<0.001$ \\
\hline Current smoking & $18.6 \%$ & $20.1 \%$ & 0.485 \\
\hline Job classification & & & $<0.001$ \\
\hline $\begin{array}{l}\text { Clerical support } \\
\text { workers }\end{array}$ & $6.9 \%$ & $31.9 \%$ & \\
\hline $\begin{array}{l}\text { Elementary } \\
\text { occupations }\end{array}$ & $10.2 \%$ & $6.3 \%$ & \\
\hline Other jobs & $46.6 \%$ & $18.9 \%$ & \\
\hline $\begin{array}{l}\text { Unemployed, home } \\
\text { makers and part-time } \\
\text { workers }\end{array}$ & $36.3 \%$ & $42.9 \%$ & \\
\hline Diabetes & $11.9 \%$ & $9.4 \%$ & 0.018 \\
\hline $\begin{array}{l}\text { Elevated waist } \\
\text { circumference }\end{array}$ & $38.6 \%$ & $39.2 \%$ & 0.793 \\
\hline Elevated fasting glucose & $36.2 \%$ & $50.3 \%$ & $<0.001$ \\
\hline Elevated blood pressure & $46.7 \%$ & $47.6 \%$ & 0.046 \\
\hline Elevated triglycerides & $39.8 \%$ & $32.1 \%$ & 0.001 \\
\hline Reduced HDL & $39.8 \%$ & $8.6 \%$ & $<0.001$ \\
\hline MetS & $36.2 \%$ & $30.5 \%$ & 0.001 \\
\hline
\end{tabular}

Mean \pm SD.

Differences in age-adjusted mean values and proportions were tested by linear regression modelling and logistic regression analysis.

*Participants who received periodontal examination and had more than 10 teeth $(n=3217$ in Korean and $n=2067$ in Japanese.

$\mathrm{CPI}$, Community Periodontal Index; HDL, high-density lipoprotein; MetS, metabolic syndrome.

influenced our findings. We have previously shown higher proportions of Prevotella and Veillonella, and lower proportions of Neisseria and Haemophilus, in the salivary microbiome of Japanese from the Hisayama study, compared with Koreans from the Yangpyeong cohort study undertaken in Yangpyeong County (a suburb of the Seoul metropolitan area).$^{20}$ Similar to our present study, the previous study showed that the periodontal condition of the Japanese was worse than that of the Koreans in the Yangpyeong cohort study, by measuring the periodontal pocket depth and clinical attachment level on mesiobuccal and mid-buccal sites of 12 specific index teeth ${ }^{20}$; 


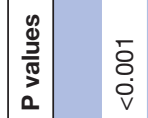

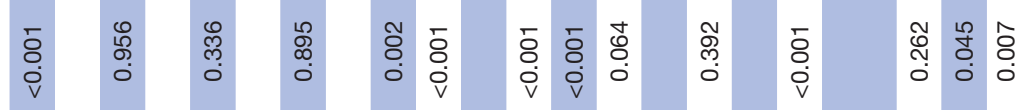

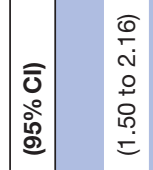

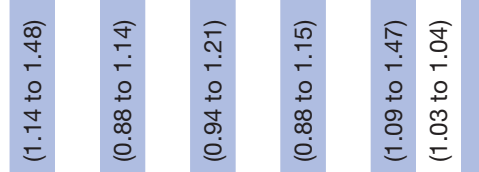

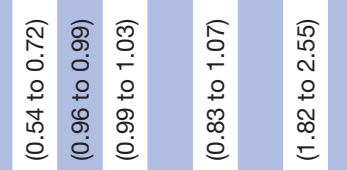

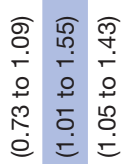

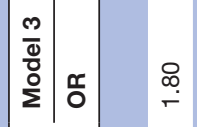

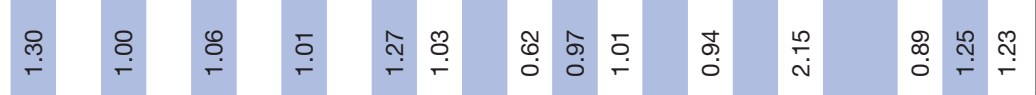

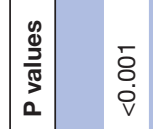

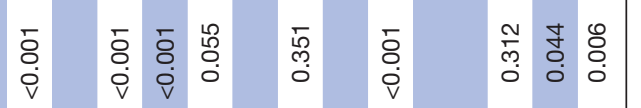

$\stackrel{0}{\circ}$

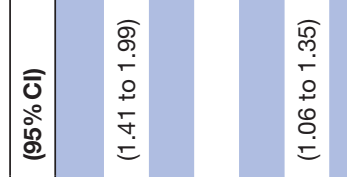

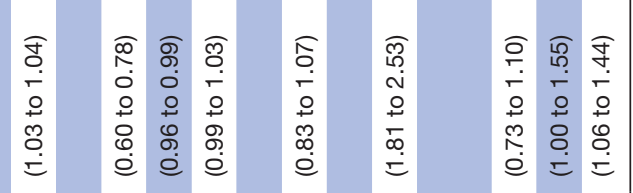

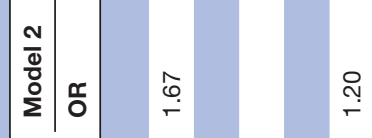

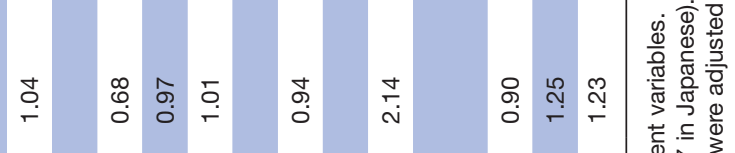

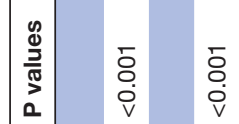

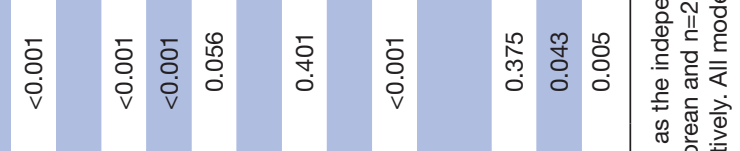

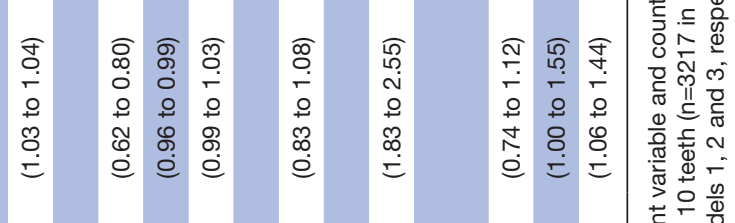

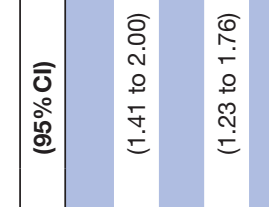

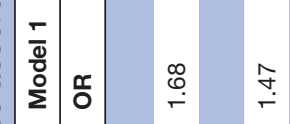

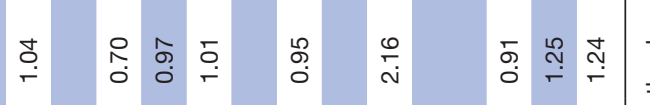

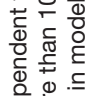


the study indicated that a greater proportion of dysbiotic oral microbiota in the Japanese is associated with a worse periodontal condition, compared with the Koreans. Thus, the difference in periodontal condition between Koreans and Japanese may be associated with disparities in their oral microbiota. It is difficult to determine the exact cause of differences in microbiomes between Koreans and Japanese. The Japanese in our previous study had a greater proportion of dysbiotic oral microbiota; this, combined with the observation that diabetes and MetS were less prevalent in the Japanese compared with the Koreans, suggests that systemic disease is less likely to be related to differences in microbiomes. Alternatively, nutrition and dietary intake might affect periodontal disease by affecting the oral bacterial community. Both Korea and Japan are based on agrarian societies and their dietary patterns are similar ${ }^{21}$; however, Koreans prefer their food spicier or saltier, compared with the Japanese. ${ }^{22}$ Kimchi, a lactic acid fermented vegetable product, is a well-known traditional Korean food. Koreans consume $96.3 \mathrm{~g}$ /day of kimchi, according to the KNHANES of $2015 .{ }^{23}$ In contrast, the Japanese consume $3.6 \mathrm{~g}$ /day of pickled vegetables, such as pickled Chinese cabbage and kimchi, based on the Japanese National Health and Nutrition Survey of $2014 .{ }^{24}$ Kimchi contains high levels of vitamins, minerals, dietary fibres and other functional components, and has been reported to have anticancer, antioxidative and antiatherosclerotic effects, as well as probiotic properties. ${ }^{25}$ Lactobacilli are responsible for kimchi fermentation. Probiotic lactobacilli have been shown to affect oral ecology by specifically preventing the adherence of other oral bacteria, and by modifying the protein composition of the salivary pellicle. ${ }^{26}$ Thus, high intake of kimchi in Koreans may be associated with a better oral environment, and a lower prevalence of periodontal disease, compared with the Japanese.

In this study, Japanese participants had a greater number of teeth than did Korean participants. Generalised linear models showed that the Japanese had a greater number of teeth ( $\beta$ coefficient $=0.19 ; 95 \%$ CI 0.17 to 0.21 ) than did Koreans, after adjusting for diabetes and covariates (online supplementary table 2). It is conceivable that the prevalence of periodontal disease was higher in Japan because of the greater number of teeth (notably, teeth with periodontal disease were not extracted in these participants). Logistic regression models showed that Japanese were more likely to have periodontal disease than were Koreans, after adjusting for the number of teeth. Additionally, when we conducted a separate analysis on the basis of the number of teeth (10-19 teeth, 20-27 teeth, and 28 and more teeth), a consistent association between country and periodontal disease was observed (online supplementary table 3 ).

Although both Korea and Japan have universal health insurance systems, the cost of dental care is lower in Japan than in Korea. Japanese health insurance covers almost all basic dental treatments such as endodontic, periodontal and prosthetic treatments including bridge and removable dentures. The cost of dental treatment in Japan is approximately $\$ 24$ (with an exchange rate of 100 yen per dollar) for resin filling in a single cavity; $\$ 60$ for infected root canal treatment in a molar with three root canals; $\$ 140$ for a gold/silver palladium crown with a metal post and core; and $\$ 26$ for molar extractions. Japanese people who receive dental treatment pay only $10 \%-30 \%$ of the total cost of treatment depending on age and income, in the universal health insurance system. In Korea, universal health insurance covers some treatments such as endodontic, periodontal, denture treatment and extraction in individuals of specific age groups. Resin filling, and prosthetic treatment, including crown and bridge, are not covered. The cost of dental treatment in Korea is approximately $\$ 100$ (with an exchange rate of 1000 won per dollar) for resin filling in a single cavity; $\$ 150$ for infected root canal treatment in a molar with three root canals; $\$ 450$ for a gold/silver palladium crown with a metal post and core; and $\$ 28$ for molar extractions. Korean people who receive infected root canal treatment and extraction pay $30 \%$ of the total cost of treatment in the universal health insurance system. This indicates that the Japanese are more likely to receive restorative and prosthetic treatment than are the Koreans, due to the lower cost of dental care. Indeed, the numbers of filled teeth (including permanent restoration by fixed dental prosthesis abutment) were higher in Japanese participants than in Korean participants in this study (table 2). Additionally, Japanese participants reported more dental visits in the last 12 months, compared with the Korean participants (table 2). The greater number of teeth in the Japanese may be attributable to the low economic barriers to obtaining dental care in Japan.

In this study, the strength of association between country and periodontal disease varied among models: the OR of country was 1.68 in model 1 , which included diabetes; 1.67 in model 2, which included MetS and 1.80 in model 3, which included individual MetS components (table 3 ). This might be due to varying associations between country and each of the following: diabetes, MetS and individual MetS components. The association between country and diabetes or MetS was not significant among participants who underwent periodontal examination and had more than 10 teeth; however, the associations between country and individual MetS components were significant (data not shown). The OR of country differed in model 3 because of interassociations among independent variables, such as country and individual MetS components.

Our study has some limitations. First, the Japanese data were derived from a single town in Japan, whereas Korean data were based on a national survey. The study cohort in Japan, which comprised residents in Hisayama town, is recognised to be demographically representative of Japan, according to the national census. ${ }^{13}$ The differences in periodontal conditions observed in this study were consistent with the reports of a national survey. Therefore, the use of study cohort in Japan to generalise 
our findings might be acceptable. Second, our findings regarding the differences in periodontal conditions between Koreans and Japanese may be affected by unmeasured factors, including oral microbiota and dietary intake. Our previous study compared the oral microbiota between Koreans and Japanese ${ }^{20}$; however, participants in Korea were drawn from a convenience sample, rather than a nationally representative sample. Future studies are needed to compare oral microbiota and dietary intake between Koreans and Japanese with data from a nationally representative sample, in order to explore the causes of differences in periodontal conditions. Third, a partial mouth assessment for periodontal condition was used. Although CPI is an easier approach to evaluate periodontal condition in the community setting, this method may inherently underestimate or overestimate the prevalence and severity of periodontal parameters due to the use of representative teeth and pseudopockets. ${ }^{27}$ Additionally, when index teeth were not present, the exclusion of sextants may have led to an underestimation of periodontal disease. In this study, all participants with more than 10 teeth had at least one sextant. The percentages of participants with 0,1 and 2-5 excluded sextants were $82.7 \%, 7.0 \%$ and $10.3 \%$ in KNHANES and $81.7 \%, 8.5 \%$ and $9.8 \%$ in the Hisayama study, respectively. Although the number of excluded sextants was relatively low, a misclassification bias may have affected the magnitude of the observed associations.

Despite these limitations, this study revealed interesting findings and has several implications. The prevalence of periodontal disease was lower in Koreans than in Japanese, whereas the prevalences of diabetes and MetS were higher in Koreans than in Japanese. These findings suggest that systemic conditions are not associated with the differences in periodontal conditions between Koreans and Japanese. We need additional studies to identify other factors that may underlie differences in periodontal conditions between countries.

\section{Author affiliations}

${ }^{1}$ Section of Preventive and Public Health Dentistry, Division of Oral Health, Growth and Development, Kyushu University, Fukuoka, Japan

${ }^{2}$ OBT Research Center, Kyushu University, Fukuoka, Japan

${ }^{3}$ Department of Preventive Dentistry and Dental Public Health, School of Dentistry, Aichi Gakuin University, Nagoya, Japan

${ }^{4}$ Department of Epidemiology and Public Health, Graduate School of Medical Sciences, Kyushu University, Fukuoka, Japan

${ }^{5}$ Center for Cohort Studies, Graduate School of Medical Sciences, Kyushu University, Fukuoka, Japan

${ }^{6}$ Department of Medicine and Clinical Science, Graduate School of Medical Sciences, Kyushu University, Fukuoka, Japan

${ }^{7}$ Department of Preventive and Public Health Dentistry, College of Dentistry, Gangneung-Wonju National University, Gangneung, South Korea

Contributors MF, YS, D-YP and YY conceived and planned the project. MF, YS, TT, JH, DY, D-YP and TN were responsible for acquisition of data. MF, KT and TT conducted statistical analysis. MF, KT and YY wrote the manuscript.

Funding This work was supported by JSPS KAKENHI Grant Number $16 \mathrm{H} 05850$ from the Ministry of Education, Science, Sports and Culture of Japan, Tokyo, Japan.

Competing interests None declared.
Patient consent Obtained.

Ethics approval This study was approved by the Kyushu University Institutional Review Board for Clinical Research (24-129).

Provenance and peer review Not commissioned; externally peer reviewed.

Data sharing statement The original data of Koreans came from Korean National Health and Nutrition Examination Survey (https://knhanes.cdc.go.kr/knhanes/eng/ index.do). No additional data are available in Hisayama study.

Open access This is an open access article distributed in accordance with the Creative Commons Attribution Non Commercial (CC BY-NC 4.0) license, which permits others to distribute, remix, adapt, build upon this work non-commercially, and license their derivative works on different terms, provided the original work is properly cited, appropriate credit is given, any changes made indicated, and the use is non-commercial. See: http://creativecommons.org/licenses/by-nc/4.0/.

\section{REFERENCES}

1. World Health Organization. An overview of CPITN data in the who global oral data bank. 2017; http://www.dent.niigata-u.ac.jp/prevent/ perio/contents.html.

2. Corbet EF, Zee KY, Lo EC. Periodontal diseases in Asia and Oceania. Periodontol 2000 2002;29:122-52.

3. Korea Centers for Disease Control and Prevention. Korean national health and examination surveys. 2018 http://cdc.go.kr/CDC/intro. html.

4. Japanese Ministory of Health Labour and Welfare. Survey of dental diseases. 2017 http://www.mhlw.go.jp/toukei/list/62-17.html (accessed 4 Aug 2017).

5. The World Bank. World bank data 2017. http://data.worldbank.org/ (accessed 4 Aug 2017).

6. Abdulla MA, Ahmed I, Assawamakin A, et al. Mapping human genetic diversity in Asia. Science 2009;326:1541-5.

7. Lee JH, Lee JS, Choi JK, et al. National dental policies and socio-demographic factors affecting changes in the incidence of periodontal treatments in Korean: A nationwide population-based retrospective cohort study from 2002-2013. BMC Oral Health 2016;16:118.

8. World Health Organization. World health statistics 2012. 2012. http:// www.who.int/gho/publications/world_health_statistics/2012/en/ (accessed 4 Aug 2017).

9. International Federation of Dental Hygienists. Working abroad as a dental hygienist. 2018;2018 http://www.ifdh.org/work abroad.html.

10. Lamster IB, Pagan M. Periodontal disease and the metabolic syndrome. Int Dent J 2017;67:67-77.

11. Levine RS. Obesity, diabetes and periodontitis--a triangular relationship? Br Dent J 2013;215:35-9.

12. Kweon S, Kim Y, Jang MJ, et al. Data resource profile: the Korea National Health and Nutrition Examination Survey (KNHANES). Int $J$ Epidemiol 2014;43:69-77.

13. Hata J, Ninomiya T, Hirakawa $Y$, et al. Secular trends in cardiovascular disease and its risk factors in Japanese: halfcentury data from the Hisayama Study (1961-2009). Circulation 2013;128:1198-205.

14. Quirynen M, Bollen CM. The influence of surface roughness and surface-free energy on supra- and subgingival plaque formation in man. A review of the literature. J Clin Periodontol 1995;22:1-14.

15. Moon JH, Koo BK, Moon MK. Optimal high-density lipoprotein cholesterol cutoff for predicting cardiovascular disease: comparison of the Korean and US national health and nutrition examination surveys. J Clin Lipidol 2015;9:334-42.

16. American Diabetes Association. Standards of medical care in diabetes--2014. Diabetes Care 2014;37 Suppl 1(Suppl 1):S14-S80.

17. Alberti KG, Eckel RH, Grundy SM, et al. Harmonizing the metabolic syndrome: a joint interim statement of the International Diabetes Federation Task Force on Epidemiology and Prevention; National Heart, Lung, and Blood Institute; American Heart Association; World Heart Federation; International Atherosclerosis Society; and International Association for the Study of Obesity. Circulation 2009;120:1640-5.

18. Hong M, Kim HY, Seok H, et al. Prevalence and risk factors of periodontitis among adults with or without diabetes mellitus. Korean J Intern Med 2016;31:910-9.

19. Lin DY, Psaty BM, Kronmal RA. Assessing the sensitivity of regression results to unmeasured confounders in observational studies. Biometrics 1998;54:948-63.

20. Takeshita T, Matsuo K, Furuta M, et al. Distinct composition of the oral indigenous microbiota in South Korean and Japanese adults. Sci Rep 2014;4:6990. 
21. Lee SI, Moon HY, Kwak JM, et al. Relationship between meat and cereal consumption and colorectal cancer in Korea and Japan. $J$ Gastroenterol Hepatol 2008;23:138-40.

22. Lee WC, Lee MJ, Kim JS, et al. Foodborne illness outbreaks in Korea and Japan studied retrospectively. J Food Prot 2001;64:899-902.

23. Park J, Lee H-J. Shifts in kimchi consumption between 2005 and 2015 by region and income level in the Korean population: Korea national health and nutrition examination survey $(2005,2015)$. Korean Journal of Community Nutrition 2017;22:145-58.
24. Japanese Ministry of Health Labour and Welfare. National health and nutrition survey. 2018; http://www.mhlw.go.jp/bunya/kenkou/kenkou eiyou_chousa.html.

25. Park KY, Jeong JK, Lee YE, et al. Health benefits of kimchi (Korean fermented vegetables) as a probiotic food. J Med Food 2014;17:6-20.

26. Stamatova I, Meurman JH. Probiotics and periodontal disease. Periodontol 2000 2009;51:141-51.

27. Kingman A, Albandar JM. Methodological aspects of epidemiological studies of periodontal diseases. Periodontol 2000 2002;29:11-30. 